\title{
Endoscopic ultrasound-guided fine-needle aspira- tion of a pulmonary artery malignant thrombus
}

Transesophageal endoscopic ultrasoundguided fine-needle aspiration (EUS-FNA) has become a standard technique for sampling mediastinal disease. The need for transvascular sampling or sampling within major mediastinal vessels seldom arises [1]. Herein, we report a case of EUS-FNA of a pulmonary artery thrombus in a patient with pancreatic carcinoma that demonstrates the impact of EUS.

A 71-year-old woman with a history of tobacco use presented with epigastric pain of 3 weeks' duration. Abdominal computed tomography revealed a $2.5-\mathrm{cm}$ mass in the pancreatic head. A tissue diagnosis was obtained by EUS-FNA that was consistent with adenocarcinoma. Initial staging by abdominal and thoracic computed tomography and fluorodeoxyglucose (FDG) positron emission tomography-computed tomography (PET-CT) demonstrated locoregional disease, in addition to a masslike region with considerably increased FDG activity in the left mediastinum and small lung nodules bilaterally ( $\bullet$ Fig. 1 ).

Because it was uncertain whether the site of primary disease was pancreatic or pulmonary, and in order to discuss the therapeutic strategy, the patient was referred to a tertiary center for EUS-FNA of the mediastinal mass. EUS was performed with a curvilinear echoendoscope (GF-UCT180; Olympus, Center Valley, Pennsylvania, USA). A pulmonary artery thrombus and a perivascular malignant-appearing process were identified ( $\bullet$ Fig. 2 ), and eight FNA passes were performed with a 22-gauge needle (Wilson-Cook Medical, WinstonSalem, North Carolina, USA) (৫ Fig.3, - Video1) via a transesophageal approach. No immediate or late adverse events were observed following EUS-FNA. The results of a preliminary on-site cytologic examination were suspicious for

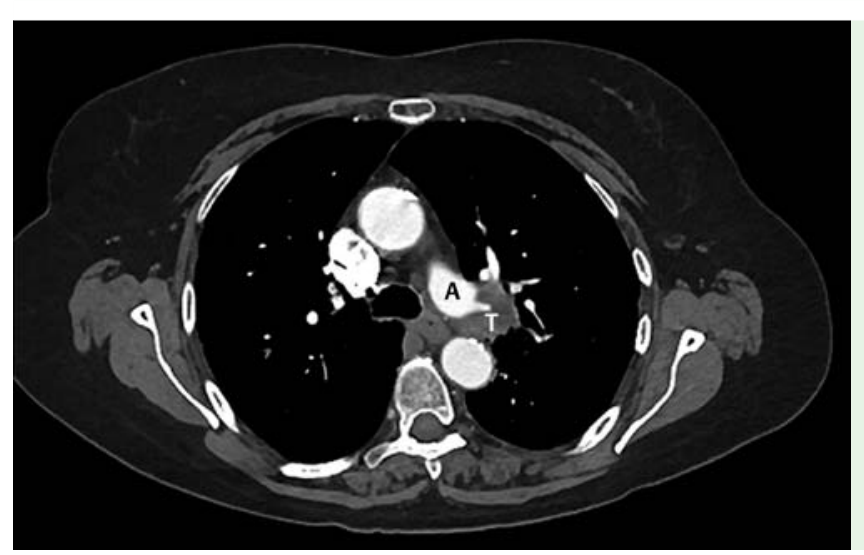

Fig. 1 Computed tomography showing a left hilar mass (T) with involvement of the pulmonary artery (A) in a 71-year-old woman with a history of tobacco use.

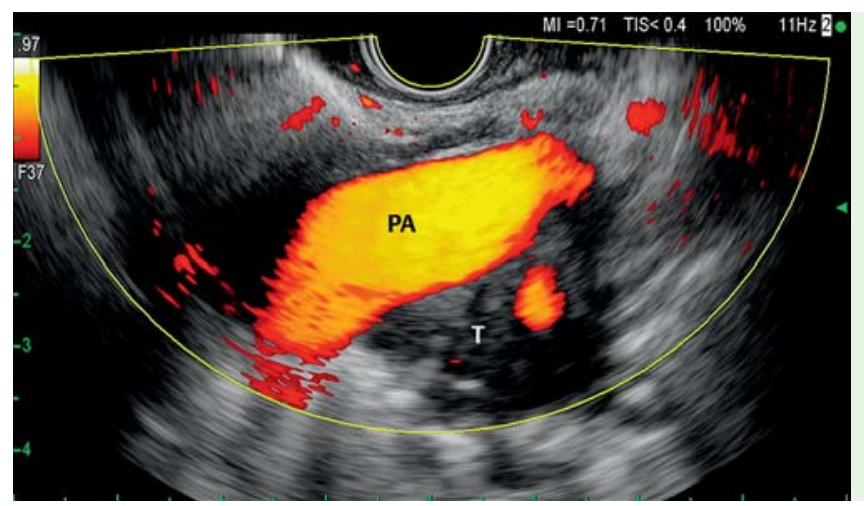

Fig. 2 Color Dopplermode endoscopic ultrasound showing the pulmonary artery (PA) thrombus ( $\mathrm{T}$ ) and left hilar mass.

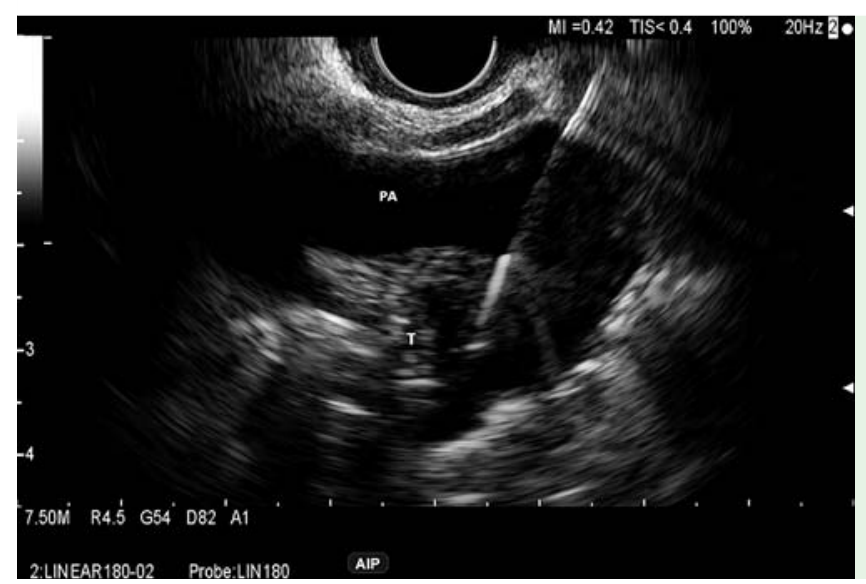

Fig. 3 Endoscopic ultrasound-guided fine-needle aspiration of a malignant pulmonary artery (PA) thrombus (T). 


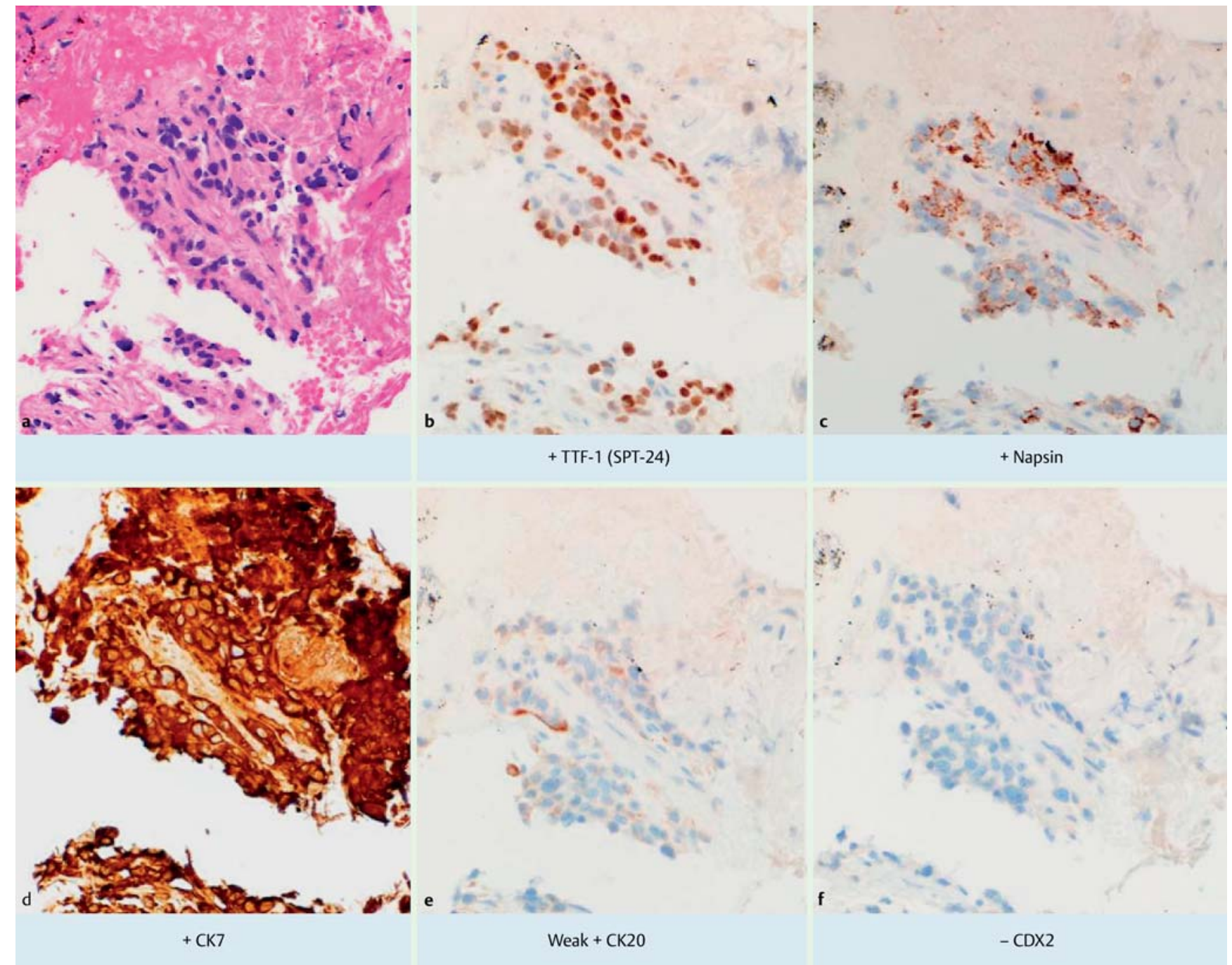

Fig. 4 Cell block from endoscopic ultrasound-guided fine-needle aspiration specimen, pulmonary artery mass. All images were acquired at $\times 400$ magnification. a Rare fragments of adenocarcinoma in a background of fibrin clot (hematoxylin and eosin [H\&E] stain). Results of tumor cell immunohistochemistry: b positivity for thyroid transcription factor 1 (TTF-1); c positivity for napsin; $\mathbf{d}$ positivity for cytokeratin 7 (CK7); e weak positivity for CK20; $\mathbf{f}$ negativity for CDX2. These findings support a diagnosis of pulmonary adenocarcinoma.

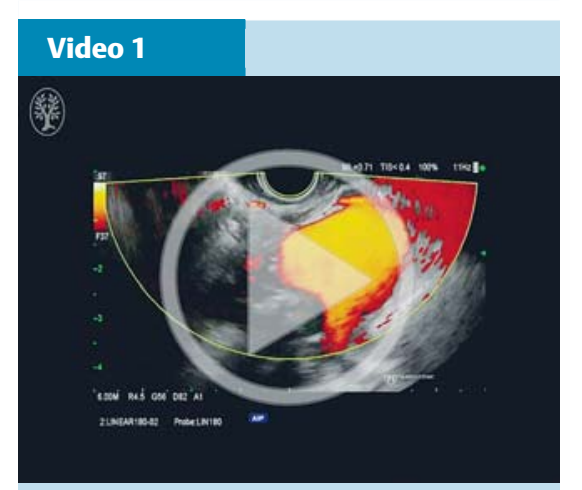

adenocarcinoma, and the final diagnosis by immunohistochemistry (IHC) was lung adenocarcinoma ( $\bullet$ Fig.4). IHC of the pancreatic tumor FNA cell block confirmed a primary pancreatic adenocarcinoma ( $\bullet$ Fig.5).

This experience further demonstrates the impact of EUS and the feasibility and potential safety of transvascular or intravas- cular EUS-FNA of major mediastinal vessels, avoiding more invasive measures and directing clinical care in carefully selected patients.

Endoscopy_UCTN_Code_TTT_1AS_2AC

Competing interests: None

Endoscopic ultrasound-guided fine-needle aspiration of a pulmonary artery malignant thrombus. 


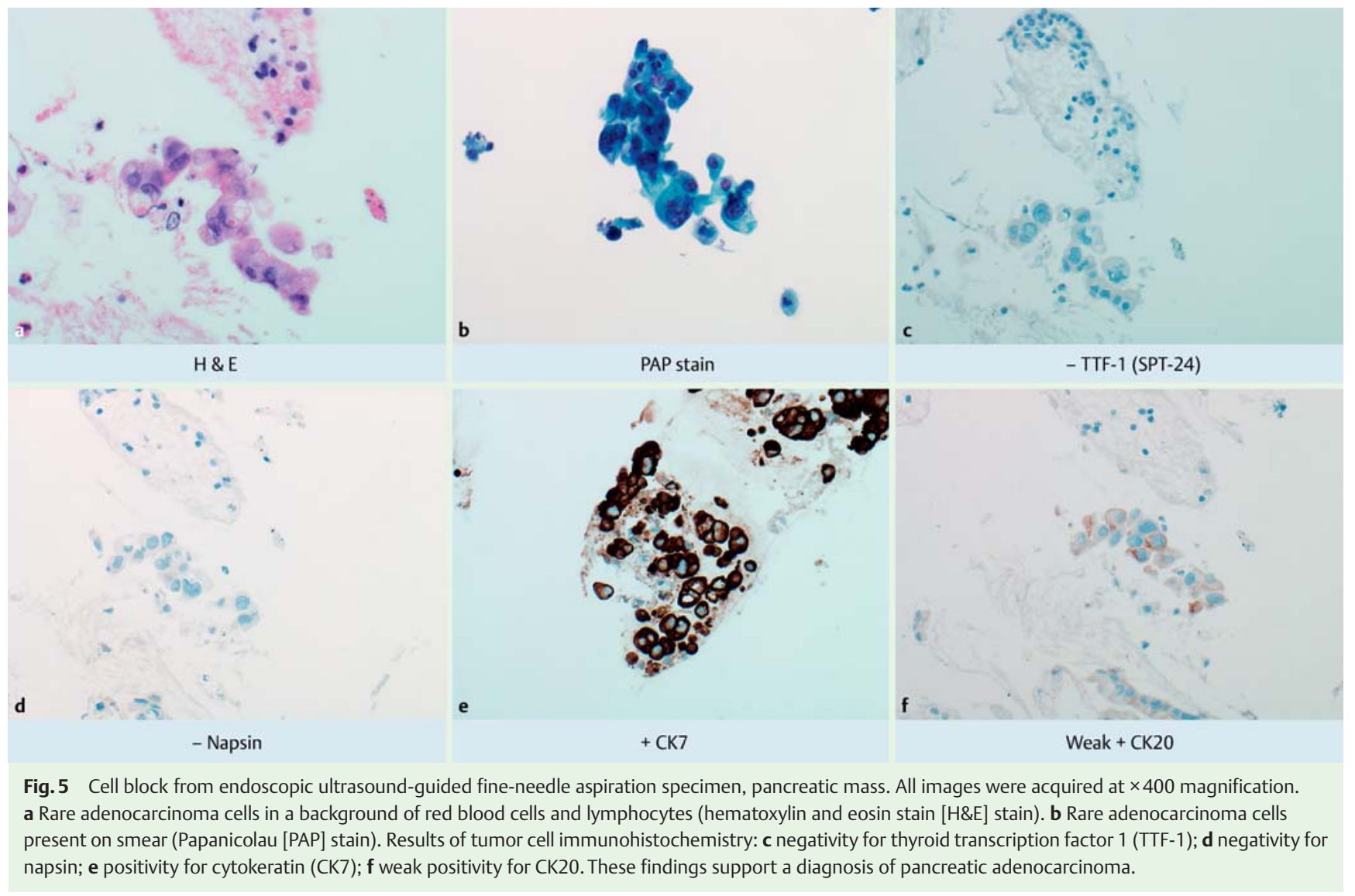

Rodica Gincul ${ }^{1,2}$, Victoria Gomez ${ }^{1}$, Sarah E. Kerr ${ }^{3}$, Jun Zhang ${ }^{3}$, Michael J. Levy ${ }^{1}$

${ }^{1}$ Gastroenterology Department, Mayo Clinic, Rochester, Minnesota, USA

${ }^{2}$ Gastroenterology Department, Edouard Herriot Hospital, Hospices Civils de Lyon, Lyon, France

${ }^{3}$ Department of Laboratory Medicine and Pathology, Mayo Clinic, Rochester, Minnesota, USA

\section{Reference}

1 Wallace $M$, Woodward A, Raimondo $M$ et al. Transaortic fine-needle aspiration of centrally located lung cancer under endoscopic ultrasound guidance: the final frontier. Ann Thorac Surg 2007; 84: 1019-1021

\section{Bibliography}

Dol http://dx.doi.org/

10.1055/s-0034-1393319

Endoscopy 2015; 47: E547-E549

(c) Georg Thieme Verlag KG

Stuttgart · New York

ISSN 0013-726X
Corresponding author

\section{Michael J. Levy, MD}

Mayo Clinic, Rochester

200 First Street SW

Rochester, MN 55905

USA

Fax: +1-507-538-5820

levy.michael@mayo.edu 